\title{
EXPLORING HOW HIERARCHICAL MODELING AND SIMULATION CAN IMPROVE ORGANIZATIONAL RESOURCING DECISIONS
}

\author{
Ericson R. Davis \\ Jeremy M. Eckhause \\ David K. Peterson \\ Michael R. Pouy \\ Stephanie M. Sigalas-Markham \\ Vitali Volovoi \\ LMI \\ 2000 Corporate Ridge \\ McLean, VA 22102, USA
}

\begin{abstract}
The resourcing environment facing businesses and governmental agencies is a complex hierarchy of interrelated decisions that span wide-ranging time horizons, where the outputs of one decision become the inputs for the next. For example, strategic resourcing decisions define multiyear, aggregate-level resource availability, which bounds the feasible region of tactical resource decisions. These tactical decisions (typically looking out over a year) disaggregate strategic resourcing decisions into a working level of resources necessary for conducting operations. Tactical decisions are themselves translated into more granular operational resource allocations. The challenge is to maintain the internal consistency of these resourcing decisions. This research describes how hierarchically integrated modeling and simulation (M\&S) techniques can assist organizations with their resourcing decisions and ensure consistency across the relevant time horizons. We demonstrate how M\&S enables a visualizing of unmanned aircraft system (UAS) employment so that support solutions can be tailored and operational effectiveness of organizational resourcing strategies can be maximized.
\end{abstract}

\section{INTRODUCTION}

The resourcing environment facing businesses and governmental agencies is a complex hierarchy of interrelated decisions that span wide-ranging time horizons. For example, strategic (long-term) resourcing plans influence decisions with a time horizon of 5 years or more, tactical (intermediate) resourcing plans span from 1 to 5 years, and operational (short-term) resource plans drive decisions within a year (Jones et al. 2000). In this resourcing environment, the outputs of one decision become the inputs for the next. Thus, strategic resourcing decisions define multiyear, aggregate-level resource availability, which, in turn, bounds the feasible region of tactical resource decisions. These tactical decisions (typically looking out over at least a year) disaggregate strategic resourcing decisions into a working level of resources necessary for conducting operations. Ultimately, tactical decisions are themselves translated into month-bymonth, week-by-week, and day-by-day operational resource allocations.

The challenge is how to maintain the internal consistency of these ever finer resourcing decisions. Of course, this is a significant task for large organizations, and they dedicate a substantial amount of energy to these resource management efforts. Consider the U.S. Army, which "implemented a broad array of complementary efforts to promote resource-informed decision making. Much work remains to inculcate 
this cost culture throughout the rest of the force, and this effort will take several years to become ingrained across the institution." (Department of the Army 2013)

This paper illustrates how a hierarchically integrated set of M\&S techniques can assist organizations with their resourcing decisions and help ensure consistency across the relevant time horizons.

\section{BACKGROUND}

Modern engineering systems have become highly complex and interrelated macrosystems-sometimes referred to as systems-of-systems - that depend on the complementary performance of their components to achieve a larger common objective. Although macrosystems enhance overall capability and functionality, they also increase the risk of subsystem failures that may cascade across the system. The consequence of such propagating failures can be widespread. The question for macrosystem designers, operators, and supportability managers becomes, how to restore a degraded or failed system to a fully operational state, especially when resources and budgets are constrained? The typical answer has been to either stock sufficient spare parts or procure enough redundant systems (e.g., airframes, vehicles, or vessels) to ensure mission capability.

Unfortunately, no single solution completely fulfills the need to balance supportability resource requirements against operational risk to achieve the requisite blend of system reliability and availability at an affordable cost. This is where hierarchically integrated sets of $M \& S$ techniques can prove useful.

\subsection{Integrative M\&S Research}

The hierarchical framework must incorporate decisions and objectives at the strategic, tactical, and operational levels. In theory, a single model that considers these different objectives could be used if such decisions are fully integrated such that the ultimate effectiveness of the operational model is incorporated into the strategic objectives. For example, Prins et al. (2007) incorporate the strategic decisions (locations) with the operational (routing) in a fully optimized way by employing a cooperative metaheuristic to solve the location-routing problem with capacitated routes and depots, but their demands and other parameters are deterministic.

Unfortunately, the performance of the operational model is rarely known with certainty, given a set of strategic decisions. Operational models must respond to immediate events that are likely not known $a$ priori. The operational model, even if deterministic over a short-time horizon, will change over time; therefore, the strategic-level decisions must incorporate that uncertainty.

Not surprisingly, there is extensive literature on optimizing strategic-level resource allocation decisions (often called facility location problems) that are responsive to the uncertainties in the reality of day-to-day operations in an integrated way. The "ambulance problem" is a classic example in which the facility decisions must respond to uncertain demand (Smoveland et al. 1973). Recent extensions of this problem include Oran et al. (2012), which considered priorities using methods that ensure higher priority locations are considered before the lower priority locations - for both facility and routing decisions. Oran et al. combined a mixed-integer program (MIP) for the facility location problem with a metaheuristic for the vehicle routing problem, which provides better performance than a maximal coverage location problem (MCLP). A standard heuristic approach is the tabu search (Glover 1990), in which heuristics are employed to escape the trap of local optimality.

Snyder (2006) provides a detailed review of different approaches currently in the literature. He outlines methods that address both stochastic optimization (maximize expected benefit) and robust optimization (minimize expected regret, or worst case scenario). While the literature is extensive on techniques, Snyder notes that relatively few methods have been applied to real-world problems, which can be attributed in part to the cumbersome data requirements. Of course, this general claim does not mean realistic problems do not exist with the framework, including problems with applications and responses to natural or manmade emergencies. For Example, Murali et al. (2012) formulate a special case of the MCLP with chance-constraints to address the demand uncertainty to provide a response to a hypothetical anthrax attack in Los Angeles County. 


\section{Davis, Eckhause, Peterson, Pouy, Siglas-Markham, and Volovoi}

In terms of tactical decisions, there has been extensive research with respect to analytical optimizations for reparable item inventories; Diaz and Fu (1995) and Guide and Srivastava (1997) provide two very detailed overviews of this area. Since World War II, reparable inventory modeling has progressed through at least five distinct phases: defining resupply pipelines, extending to multi-item optimization, introducing multi-echelon considerations, linking investment to readiness, and explicitly considering enditem complexity. (O'Malley and Peterson 2010) While all of these phases affect the hierarchical resourcing decision, this paper specifically focuses on the inventory performance aspects.

Since the 1960s, as inventory modeling research matured, the optimization goals for measuring reparable inventory system performance evolved from item-level fill rates and backorders, through systemlevel expected backorders, and ultimately to system-level operational availability. O'Malley et al. (2012) provide a more detailed review of reparable inventory performance measures, but today's state of the art optimizes reparable item inventory levels toward achieving an end-item availability goal. Of course, analytical optimizations do make assumptions about demand rates and distributions, lead times, end-item usage rates, and the like. Testing the robustness of these assumptions, and translating availability into operationally meaningful measures, is where simulation techniques come into play.

Paralleling the evolution of reparable inventory modeling was a complementary effort in inventory system simulation. Banks and Malave (1984), in their survey of inventory system simulations, developed a six-category classification schema to describe how simulation can complement inventory modeling. One of their categories was "verification of analytic solutions," which is a natural complement to tacticallevel inventory optimizations. In fact, over the years, simulations have frequently been used to operationally evaluate recommended inventory solutions. For example, Smith et al. (2006) described how they developed a discrete event simulation (the Joint Strike Fighter Support Enterprise Model) to provide "logistics analysts with the ability to define an operational and support environment and ascertain measures of [the inventory system's] performance effectiveness." These performance measures included statistics such as aircraft availability and flying hours.

In a similar vein, Siddiqi and de Weck (2007) developed a two-stage approach. The first stage was an availability-based, analytical sparing model for "estimating requirements of reconfigurable spare parts." In the second stage, the validity of this solution was tested using a discrete event simulation to estimate the availability of a space exploration hardware system when resupply was unavailable. Another example of integrating analytical and simulation methods is from Klingebiel and Li (2011), who used an integer programming approach to develop optimal sparing policies for a multiechelon inventory system. They then used a simulation to "test the robustness of the 'optimal' inventory policies generated from the analytical model..." Finally, some integrated multiechelon analytical sparing models have been linked to reliability and maintainability models, then implemented as routine practice. For example, the Navy's Availability Centered Inventory Model and TIGER simulation ties sparing allowances and wholesale stock level decision models to the availability of shipboard systems. (OPNAV 2000)

Clearly, the integration of analytical inventory optimizations with discrete event simulations is a longestablished best practice within the logistics community. Our work differs from most of this literature in two ways. First, to the best of our knowledge, the integration of network design (facilities), inventory optimization at the tactical level, and system performance at the operational level (which requires the integration of three layers of models) has not been done for real-world problems related to UAS resourcing. Indeed, Snyder's (2006) review of the state of the art identifies a need to capture the costs of tactical and operational models of the supply chain under uncertainty. In this paper, we provide an example that demonstrates the benefits of this integration.

Second, the integration of models whose performance cannot be described with simple parameters or expected values makes most of the elegant models described in Snyder (2006) inapplicable. This issue does not mean that existing methods are not available; tabu searches and other metaheuristics can still be incorporated to provide reasonable solutions when the lower-level simulation model's performance is difficult to describe parametrically.

For this work, we optimize based on the expected reward according to the historic demand. This type of objective is admittedly an approximation of the expected performance of a simulation-based operation- 
al model. The full integration of these models ultimately would be improved by exploring the tabu searches and other heuristics previously noted.

\subsection{An Application Scenario and the Hierarchically Integrated M\&S Framework}

In 2012, a law was implemented instructing the Federal Aviation Administration (FAA) to rewrite regulations and develop a strategy for expanding the use of UASs in the United States. The FAA Modernization and Reform Act of 2012 directed the FAA to fully integrate unmanned systems into the national airspace by 2015. This tasking was a direct outcome from the exponential growth in federal (both Department of Defense and non-DoD) UAS domestic operations. While the DoD's domestic use of UASs (e.g., training, research and development, and capabilities development) is well-understood, UAS usage by federal agencies is less well known and even more diverse. For example, federal agencies are using UASs for everything from law enforcement to forest management, and from climatological investigations to geological surveys.

The rapid expansion of UAS usage by federal agencies has been facilitated, at least in part, by the military services' resizing of their UAS fleets to fit within the anticipated post-Iraq and Afghanistan force structure. Many small- and mid-sized UASs (and their support equipment) are being made available to federal agencies. Of course, these "free" UASs can be both a blessing and a curse. While federal agencies have become very skilled at repurposing former military UAS systems for non-defense missions, these same agencies now need to develop (and fund) a UAS support infrastructure to sustain their UAS missions and select an appropriate operations and support (O\&S) strategy. (It appears that agencies are pursuing a variety of UAS O\&S strategies, ranging from government-owned, government-operated to contractor-owned, contractor-operated and everything in between.)

Given the current need within the federal UAS community to better quantify and document UAS program costs and resourcing, we believe a hierarchical M\&S framework can help federal agencies integrate the myriad business resourcing decisions they face as the systems are deployed within their federally authorized charters. These difficult, multifaceted resourcing decisions warrant a sophisticated M\&S approach to organizational decision making.

In this paper, we propose a hierarchical analytical framework to assist with structuring the organization's M\&S efforts with respect to resourcing decisions. This hierarchical M\&S framework is expressly designed to support multi-faceted decisions (see Figure 1). The iterative framework uses optimization and simulation techniques to model operational and logistical networks.

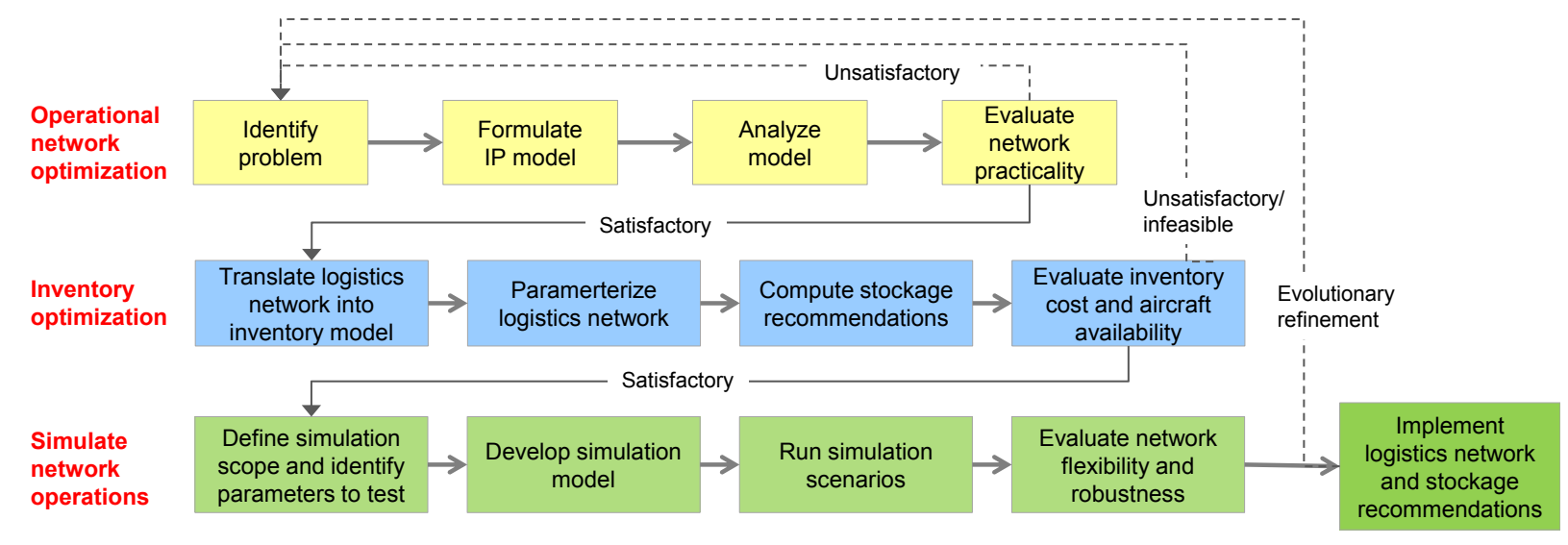

Figure 1: The Hierarchically Integrated M\&S Framework.

\section{METHODOLOGY}

We consider a MQ-1 Predator UAS unit deployed to observe and report wildfire activity within a selected geographic region. The MQ-1s are operated from select bases servicing specified orbital areas located as 
to maximize the MQ-1s' surveillance coverage over areas of high fire risk along a wildland-urban interface. The MQ-1 bases will be resourced to be self-sufficient for 30 days of deployed operations, but they can also be supported from higher echelon logistics facilities (e.g., depots and manufacturers) if required (or desired).

\subsection{Strategic-Level Decisions}

Two strategic-level resourcing decisions need to be made in this scenario: how many UASs are needed, and where should they be located? With finite resources and a limited number of available UASs, it is desirable to position them to achieve the greatest impact with regard to wildfire monitoring. Given the stochastic nature of fire outbreaks and the uncertainty associated with the time to discover the outbreak, a deterministic optimization is, at best, an approximation of the probabilistic outcomes. Indeed, the system performance at the operational level is the ultimate measure of effectiveness. Nevertheless, for strategiclevel planning, an objective function based on a static reward structure, such as a heat map, could create a reasonably desirable set of outcomes.

The value of monitoring one area for wildfires is not the same as the value of monitoring other, equally sized areas. For example, a fire could spread quickly in a dense boreal forest, whereas in a steppe land, a wildfire is either unlikely or easily managed; therefore, expending resources to monitor the forest would be considered of greater "value." Moreover, regions with higher population densities are intrinsically more valuable because of the higher probability of loss of life or property.

\subsubsection{Reward Heat Map}

We divide a region of interest into discrete grid squares of approximately 4 square miles. For the grid space at coordinate (i,j), we quantify the value $r_{i j}$ associated with monitoring that space in the following way:

$$
r_{i j}=\alpha F(i, j) *(1-\alpha) D(i, j, \sigma) \text {. }
$$

$F(i, j)$ is the percentage of forest coverage of grid space at $(i, j)$. $D(i, j, \sigma)$ is the Gaussian smoothed population density (smoothing parameter $\sigma$ ), and $\alpha \in[0,1]$. Population density and forest coverage information were developed from vegetation (CSFS 2012) and population (Wikimedia Commons 2012) images.

\subsubsection{Integer Program Model}

In our strategic level planning, we position each UAS at a site chosen from a fixed list of predetermined sites, typically existing airports. Since there is little value in visiting a single grid space multiple times in rapid succession, the marginal benefit achieved by adding aircraft to a given site will decrease with each additional aircraft. Each candidate location has a set of grid spaces that a UAS, leaving from that location, can visit and return from in a single sortie. We define $A$ for each grid square, airport location, and a given number of UASs, as $A_{i j k} \ell$, or the percentage of grid square $(i, j)$ that is covered if there are $\ell$ UASs stationed at location k. $A_{i j k}$ is based on the performance characteristics (range, speed, etc.) of the UASs. With $A$ defined thusly, we can generate an integer program (IP) that maximizes the coverage given a fixed number of aircraft and available locations (i.e., airports) as follows:

$$
\begin{aligned}
& \operatorname{Max} \sum_{i} \sum_{j} r_{i j} g_{i j} \\
& \text { s.t. } g_{i j} \leq \sum_{k} \sum_{\ell} A_{i j k l \ell} Z_{k \ell} \quad \forall k, \ell \\
& \begin{array}{l}
\sum_{\ell} z_{k \ell}=1 \\
\sum_{k} \sum_{\ell} c_{k \ell} z_{k \ell} \leq B
\end{array}
\end{aligned}
$$


Davis, Eckhause, Peterson, Pouy, Siglas-Markham, and Volovoi

$$
z \in\{0,1\} \text { and } 0 \leq g_{i j} \leq 1
$$

where $\mathrm{g}_{i j}$ is the fraction of coverage for grid square $(\mathrm{i}, \mathrm{j}), \mathrm{z}_{k} \notin$ is a binary decision variable ( 1 if there are $\ell$ UASs at location $\mathrm{k}, 0$ otherwise), $\mathrm{c}_{k}$ is the cost of positioning $\ell$ UASs at location $\mathrm{k}$, and $\mathrm{B}$ is the total budget.

In this IP formulation, we assume the reward $\mathrm{r}_{i j}$ is weighted by the region of interest based on population and wildfire risk. Of course, in reality, the fires and their potential damage are stochastic events, and the UAS' ultimate effectiveness is determined by the operational models within this hierarchy. Nevertheless, this strategic-level decision, as determined by the IP, will affect the performance at the operational level.

\subsection{Tactical-Level Decisions}

The tactical resourcing decision to be considered in this scenario is the level of inventory investment needed to support the planned UAS operations to achieve a desired level of system availability? (We only consider sparing the MQ-1 aircraft to the operating locations selected in strategic level decision.)

Modern aerospace systems are increasingly complex and interrelated. These macrosystems rely heavily on the complementary performance of their constituent systems to achieve a larger common objective. A fully operational MQ-1 unit consists of multiple aircraft, ground control systems (GCSs), and ground data terminals (GDTs) (Figure 2). The MQ-1 depends on the complementary performance of both its airborne and ground-based components to fulfill its missions.

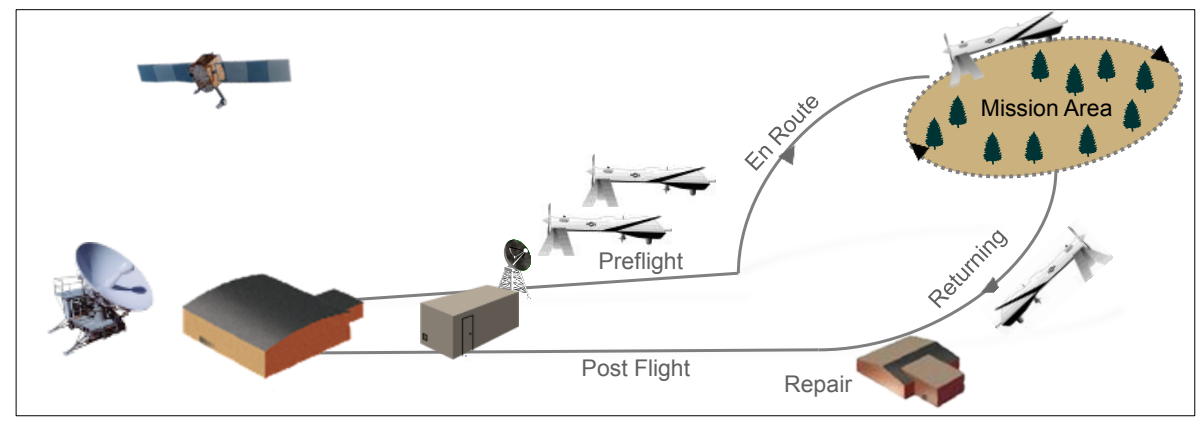

Figure 2: A Notional MQ-1 UAS Wildfire Deployment Scenario.

A readiness-based sparing (RBS) inventory optimization method is used to compute the spares investment requirements for the MQ-1 deployment given the strategic-level basing decisions. RBS techniques view an MQ-1 as a composite of its major components (sensors, electronics, propulsion, structural components, etc.).

We employ LMI's Aircraft Sustainability Model $^{\circledR}\left(\mathrm{ASM}^{\circledR}\right)$ sparing model (Aircraft Sustainability Model and ASM are registered trademarks of the Logistics Management Institute), a well-known RBS application, to compute the MQ-1 reparable spares requirements. The ASM model's replenishment pipelines use operating scenario characteristics in conjunction with typical component-level data (demand rates, maintenance and transportation times, and costs). ASM then employs marginal analysis to rank possible additions to the spares inventory in terms of their availability benefit per dollar, thereby guaranteeing an efficient and effective spares mix. Accumulated costs and aircraft availabilities are tracked as the spares list is developed to create a curve that relates inventory investment (the x axis) to system availability (the y axis). Logistics planners use these traditional availability-to-cost curves to formulate budgets, allocate resources, and identify spares purchases.

\subsection{Operational-Level Decisions}

Once the strategic- and tactical-level resourcing decisions are made, we evaluate the robustness of these decisions in the face of day-to-day operational variability. Simulation-based methods are well suited to this task, as they can readily capture the complexity, interdependencies, and variability inherent in a mac- 
rosystem's O\&S. To illustrate this portion of the hierarchical M\&S framework, we employ a reliability and maintainability (R\&M) simulation and an agent-based model (ABM).

\subsubsection{R\&M Modeling}

While RBS methods can assess the availability of individual subsystems, they cannot fully assess the probability of a macrosystem achieving its objective. For example, "readiness" describes a system's availability to perform its mission, but readiness is only one of several factors that ultimately lead to mission success. To truly assess a macrosystem's mission performance, we combine RBS analysis with modern simulation tools. We use the Stochastic Petri Nets with Aging Tokens (SPN@) R\&M software to model the UAS system-of-systems. (Volovoi 2006)

SPN@ is used to model the MQ-1 wildfire surveillance mission cycle and serves as the framework for adding macrosystem complexities to the model. For example, a local GCS and GDT are both required for the MQ-1 aircraft to fly. Once the aircraft is launched and en route, these local ground systems hand off control to a non-local GCS. If either, or both, of the local ground system components are inoperative, then the mission may be compromised or prematurely curtailed - thereby diminishing the percentage of time wildfire surveillance coverage can be maintained.

Figure 3 illustrates the SPN@ simulation. This model describes the MQ-1 mission, from the pool of available aircraft, through a preflight check and take-off. After transiting to the mission area, the wildfire surveillance begins. At some point, the MQ-1 must call for a relief aircraft to continue the surveillance after the original aircraft departs the mission area and returns to base for a post-flight check and any requisite corrective maintenance. Supporting this mission are the line-of-sight (LOS) ground-based subsystems (the GCS and GDT), which must be fully operational for MQ-1s to take off and land.

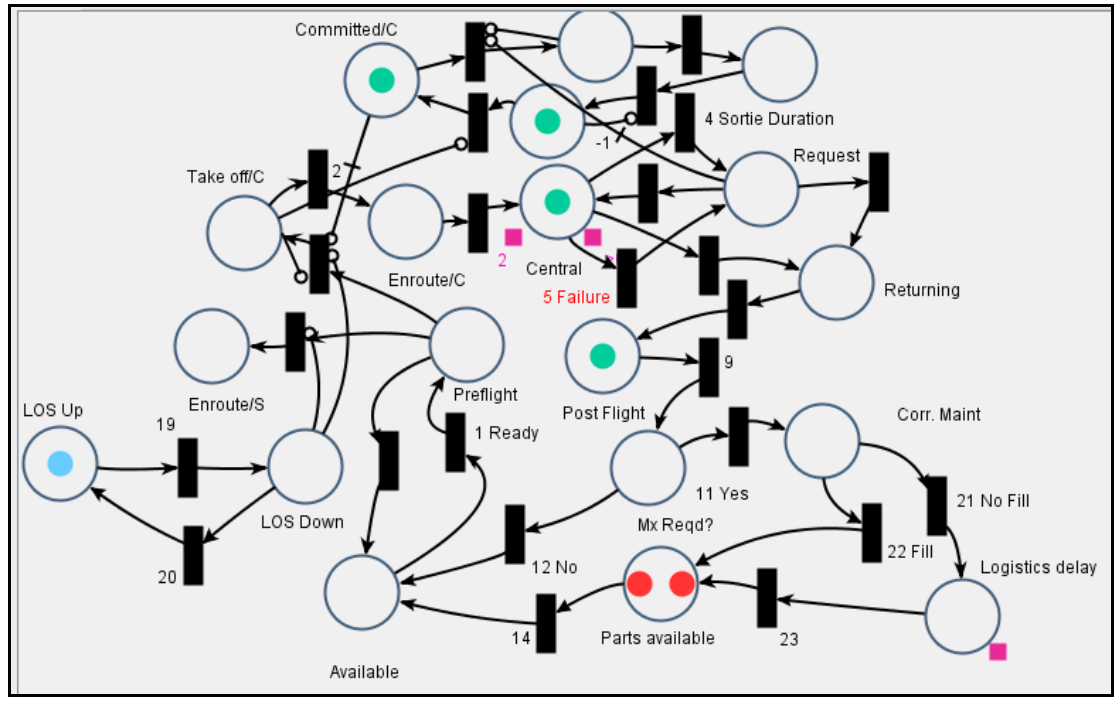

Figure 3: Example of SPN@ Wildfire Mission Model.

The use of Stochastic Petri Nets (SPN) enhances the fidelity of modeling the MQ-1 mission cycle by enabling explicit consideration of key logistical realities (e.g., a corrective maintenance cycle, spares availability, and logistical delays). SPN provides a "local" representation of the macrosystem by modeling its constituent systems and the rules governing their interdependencies. The localized nature of a SPN representation dramatically improves the scalability of macrosystem models by mitigating the combinatorial "curse of dimensionality." In addition, SPN's employment of Monte Carlo simulation allows us to relax Markovian assumptions and account for dynamic effects.

For the MQ-1 UAS model, using aging tokens in SPN@ enhances modeling power by capturing timevarying performance nuances, which offers a unique way to model the aging processes without sacrificing the dynamic features of SPN modeling. For example, the green tokens in Figure 3 represent fully servicea- 
ble MQ-1s that are either performing their mission or are mission ready; the red tokens represent MQ-1s that are grounded as a result of component failures and logistical delays. Thus, the SPN@ model enables us to account for the effects of in-flight failures as well as delays associated with maintenance activities.

In addition, unlike standard discrete-event simulation tools, SPN@ graphically models system failure dependencies and their propagation. Moving tokens visually represent the system dynamics associated with those failures.

\subsubsection{ABM Simulation}

While the SPN@MQ-1 simulation measures the percentage of the time the MQ-1s can maintain their round-the-clock wildfire surveillance coverage, it cannot estimate the effectiveness of MQ-1s in the timely detection of the wildfires. To make this measurement, we turn to an agent-based model. Our ABM uses a fairly simple fire spread model with fixed barrier patrol (Champagne et al. 2003) search patterns for the UASs. (See Vincent and Rubin [2004] and Choset [2001] for descriptions of alternative UAV search patterns). Throughout the simulation, each grid space $(\mathrm{i}, \mathrm{j})$ is capable of spontaneously catching fire (e.g., from a lightning strike) with probability $\mathrm{p}(\mathrm{i}, \mathrm{j}, \mathrm{t})$, where $\mathrm{p}$ is a function of time $\mathrm{t}$. In this way we can model semi-random lightning strikes throughout the region as a storm (or series of storms) sweeps from west to east.

Once a grid space has ignited, its immediate neighbors are exposed to the risk of catching fire. A grid space $(i, j)$ with a burning neighbor will begin to burn with a probability given by $n(i, j, v)$, where $v$ is the wind vector). Once a grid space ignites, it will burn for an exponentially distributed duration until it is extinguished. Once extinguished, it cannot reignite. While this is occurring, the UASs are orbiting the region looking for any burning grid spaces. When a UAS passes within a sensor swath width of a burning space, we assume that the fire in that grid space is reported immediately.

The output of the ABM is a list of which grid spaces were reported as burning during the trial. Using this list, we calculate the final "score" using the total of all the burned squares. Of course, a single trial isn't sufficient so we compile statistics across 1000 trials to gauge operational success.

\section{DISCUSSION}

Through the use of integrated M\&S techniques, federal agencies can quantify the resources required to sustain their UAS strategies. This fits well with a hierarchical decision framework, as we demonstrate with notional UAS deployment decisions that have distinct strategic, tactical and operational aspects.

The MQ-1 case study illustrates the possible hierarchical integration of a combination of analytical optimizations with simulations, and the scenario demonstrates the utility of this approach. The hierarchical decision framework evaluates the mission coverage that can be achieved as we consider investment trade-offs in spares, additional aircraft, and deployed support capabilities.

At the strategic level, the number and deployment locations of the MQ-1s are significantly influenced by the desired performance goals (in this case, to maximize surveillance coverage of high-risk wildlandurban interface areas). Several factors constrain the number and location of the deployed MQ-1s, including qualified candidate airports and the total cost of airport basing, maintenance, and flying operations.

By combining forest coverage and population density data, we allow different reward values for regions in the potential orbital areas; thus, some regions will be visited more often than others. Applying the IP solution results in a recommended deployment of MQ-1s to northern, central, and southern orbital locations. Figure 4 shows the location of each airport and the approximate coverage areas (assuming a 10hour sortie). We note that the coverage is greatest (where the orbits overlap) in the most population dense wildland-urban interface areas. 


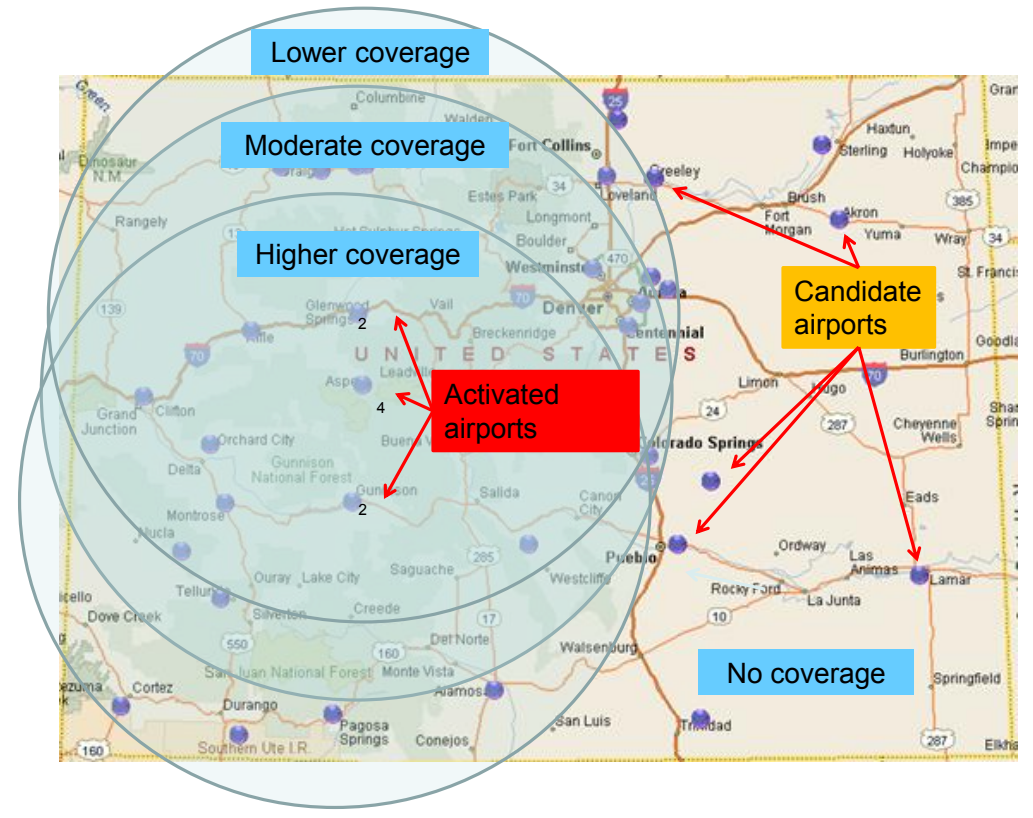

Figure 4: Proposed MQ-1 Orbital Locations.

At the tactical level, spares range and depth decisions are influenced by the UAS fleet size and location. In our scenario, we model the MQ-1 deployment of eight aircraft allocated as shown in Figure 4. The goal is to generate the equivalent of 10 hours per aircraft per day with an 80 percent operational availability across the deployed aircraft. Each aircraft is modeled as a composite of its corresponding subsystems (e.g., fuel, engine, structural, electronics, etc.), all of which have unique costs, repair times, and failure rates. To provide a more conservative sparing requirement, we start with some typical inventory assumptions for DoD systems, such as no resupply for the first 30 days of the deployment and no parts cannibalization between aircraft. The ASM sparing model, which is used to perform the RBS analytical optimization, recommends an inventory investment of $\$ 17.6$ million. At the given operational tempo, this level of inventory would support an 82 percent operational availability, and would immediately satisfy 87.7 percent of anticipated spares requests. Any spares requests not filled immediately would experience a 3.3-day logistics delay (once resupply is allowed).

At the operational level, it is essential to understand the logistics network's robustness for day-to-day operational variability, so we transition from analytical models to discrete event simulations. Simulations enable us to better portray the vagaries of daily flight operations and capture their effect on wildfire surveillance. We model the eight aircraft, which are operated out of three airfields and begin their surveillance orbits immediately upon takeoff. We further assume that, at any given time, the desire is to have one-half of the aircraft assigned to the northern (1 out of 2), central (2 out of 4), and southern (1 out of 2) orbits in the air surveilling, while the remaining aircraft are at home station being maintained and readied for their next mission.

Within the SPN@ operational simulation, the LOS ground-based subsystems are modeled as having a 95 percent uptime and a 5-hour mean time to repair, while the aircraft have a 77 hour mean time between failure and a 14.6 hour mean time to repair. (For both the aircraft and ground-based subsystems, we assume the time to failure is exponentially distributed.) From the SPN@ simulation, given the preceding inventory investment decisions, the percentage of time that MQ-1s are orbiting the northern, central and southern regions are 91.9, 91.7, and 91.9 percent respectively. Assuming 100 percent orbital coverage is too optimistic, and the impact of operational and logistical variability must be taken into account when planning the MQ-1 orbital search patterns.

The ABM simulation allows us to extend this greater degree of operational realism to planning the MQ-1 wildfire surveillance search patterns. Specifically, to illustrate the importance of this consideration, 
we modeled the northern, central, and southern orbital areas within an ABM where a fire is randomly initiated and results in a risk of spreading to adjacent cells if the fire is not discovered and extinguished quickly. Each replication of the simulation represents 34.7 days of the wildfire season.

In the first run of the ABM model, we employ no MQ-1s to perform aerial wildfire surveillance and measure the amount of damage to high-value wildland-urban interface areas. Under this scenario, 4.8 percent of the forested areas are destroyed on average. In the next run, the MQ-1s are deployed. In this scenario, an average of 2.1 percent wildland and property are destroyed — a net reduction of 2.7 percent from the previous scenario.

Figure 5 illustrates the effectiveness of employing MQ-1s to spot wildfires. Specifically, it shows that the forested area that is burned is dramatically reduced due to the MQ-1s spotting and reporting wildfires earlier, before they have a chance to spread.

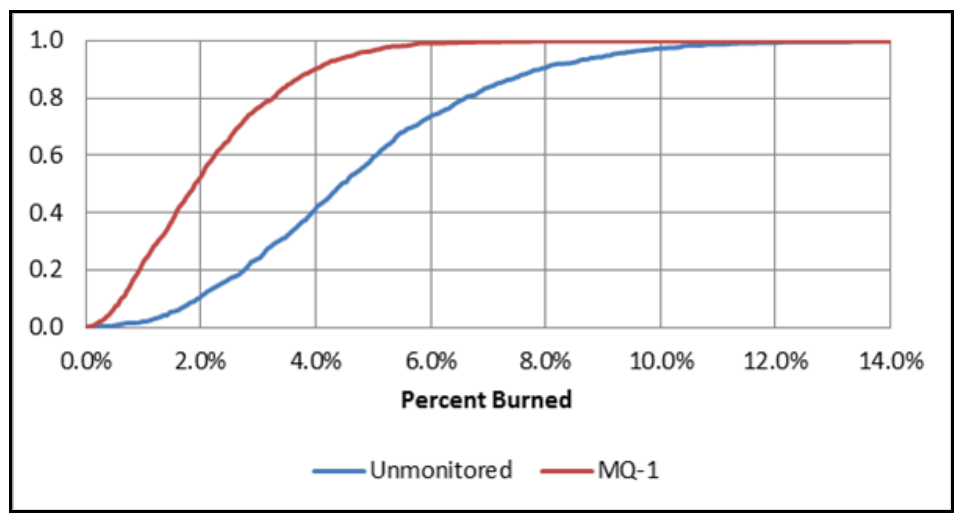

Figure 5: The Impact of MQ-1 Surveillance (Described by CMFs).

According to a 2010 report by the Western Forestry Leadership Coalition, five large wildfires between 2000-2003 burned more than 882,000 acres and resulted in \$2.9 billion in damage (Dale 2010). The average cost per acre burned was more than $\$ 3,300$, recognizing, of course, that the type of land and level of development varied significantly from area to area and fire to fire. Being able to estimate the typical cost of lost wildland and structures allows us to extrapolate the potential value of the MQ-1s' early wildfire detection and the opportunity for suppression. Knowing this provides a valuable comparison against the operational and logistical costs of deploying the MQ-s, as determined through the hierarchically integrated resourcing decision process.

\section{SUMMARY}

The proposed hierarchically integrated M\&S methodology offers macrosystem designers, operators, and supportability managers a pragmatic and effective approach to leverage the power of complementary analytical techniques for coping with the ever-more complex support requirements of modern macrosystems.

\subsection{The Value of Hierarchically Integrated M\&S}

An integrated approach is essential to achieve "good," if not optimal, performance in a macrosystem hierarchical model. Even the best solution techniques will only achieve locally optimized solutions if the ultimate performance of the operational model is not addressed in the strategic-level decisions.

We implemented a hierarchically integrated resourcing model, albeit on a limited scale. Our strategiclevel IP solves the facility location problem with the goal of maximizing ultimate system performance at the operational level. However, while the implementation of a strategic-level optimization (which optimizes according to an approximation of the ultimate performance) is an important first step, that solution must be examined for its overall effectiveness. 
Davis, Eckhause, Peterson, Pouy, Siglas-Markham, and Volovoi

\subsection{Future Research}

As noted above, there is considerable literature on the techniques to improve strategic-level decisions to optimize operational-level performance. Tabu searches and other metaheuristics can be implemented successfully, especially if the lower-level simulation model can generate results quickly and iteratively. Even without a fast-time simulation, evaluating a set of strategic-level optimizations could improve the performance and robustness of the solution, though formal bounds on optimality are unlikely.

We wish to pursue several ABM enhancements. The first is to fine tune the ABM's calibration in terms of event speed. Another area for further research is in the measuring of the aerial surveillance's effectiveness; it would be preferable to deploy the IP heat map into the ABM and record a measure of relative rewards. We also wish to enhance the ABM model with "intelligent" actors and make the ABM sufficiently robust that it can serve as a platform for testing the relative effectiveness of alternative search patterns or algorithms while incorporating more complex topographical and environmental factors. Finally, we will explore possible extensions to this problem set, such as using UASs to find active hiders (e.g., poachers), or applications for other systems-of-systems, such as NOAA's tsunami warning system.

\section{REFERENCES}

Banks, J. and C. O. Malave. 1984. "The Simulation of Inventory Systems: An Overview." Simulation. 42(6), 283-290.

Champagne, L., R. G. Carl, and R. Hill. 2003. "Search Theory, Agent-Based Simulation, and U-Boats in the Bay of Biscay." In Proceedings of the 2003 Winter Simulation Conference, Edited by S. Chick, P. J. Sánchez, D. Ferrin, and D. J. Morrice, 991-998. Piscataway, New Jersey: Institute of Electrical and Electronics Engineers, Inc.

Choset, H., 2001. "Coverage for robotics - A survey of recent results." Annals of Mathematics and Artificial Intelligence. 31(1-4), 113-126.

Colorado State Forest Service (CSFS), "Vegetation Map." Accessed 30 July 2012. http://csfs.colostate.edu/pdfs/veg-map-web.pdf.

Dale, Lisa. 2010. "The True Cost of Wildfire in the Western U.S." Western Forestry Leadership Coalition. Accessed March 24, 2013. http://www.wflccenter.org/news_pdf/324_pdf.pdf.

Department of the Army, "2013 Annual Report on Business Transformation Providing Readiness at Best Value." Accessed March 15, 2013. http://www.army.mil/standto/archive_2013-03-15/?s_cid=standto.

Diaz, A. and M. C. Fu. 1995. "Multi-Echelon Models for Repairable Items: A Review." Working Paper, University of Maryland.

Glover, F. 1990. "Tabu Search: A Tutorial,” Interfaces, 20(4): 74-94.

Guide, V. D. R., Jr. and R. Srivastava. 1997. "Repairable Inventory Theory: Models and Applications," European Journal of Operational Research, 102(1): 1-20.

Jones, G. R., J. M. George, and C. W. L. Hill. 2000. Contemporary Management, $2^{\text {nd }}$ ed. Boston, Massachusetts: Irwin, McGraw Hill.

Klingebiel, K. and C. Li. 2011. "Optimized Multi-Echelon Inventory Policies in Robust Distribution Networks. In: Proceedings of 25th European Conference on Modeling and Simulation, edited by T. Burczynski, J. Kolodziej, A. Byrski, and M. Carvalho, 573-579. Krakow, Poland, ECMS.

Murali, M., F. Ordonez, and M. M. Dessouky. 2012. "Facility Location under Demand Uncertainty: Response to a Large-Scale Bio-Terror Attack," Social-Economic Planning Sciences, 46(1): 78-87.

O'Malley, T. J. and D. K. Peterson. 2010. "The Evolution of Readiness-Based Sparing Models," Logistics Spectrum, 44(2), 17-21.

O’Malley, T. J., R. D. Moulder, and D. K. Peterson. 2012. "Sizing Inventories with Readiness-Based Sparing," Air Force Journal of Logistics, XXXV(3 and 4), 18-27.

OPNAV. 2000. OPNAV Instruction 4442.5: Readiness Based Sparing. Department of the Navy, Washington, DC, June. 
Oran, A., K. C. Tan, B. H. Ooi, M. Sim, and P. Jaillet. 2012. "Location and Routing Models for Emergency Response Plans with Priorities," Comm. in Computer and Information Science, 318, 129-140.

Prins, C., C. Prodhon, A. Ruiz, P. Soriano, and R. W. Calvo. 2007. "Solving the Capacitated LocationRouting Problem by a Cooperative Lagrangean Relaxation-Granular Tabu Search Heuristic," Transportation Science, 41(4): 470-483.

Siddiqi, A. and O. L. deWeck, 2007. "Spare Parts Requirements for Space Missions with Reconfigurability and Commonality." Journal of Spacecraft and Rockets. 44(1): 147-155.

Smith, V. D., D. G. Searles, and B. M. Thompson. 2006. "SEM: Enterprise Modeling of JSF Global Sustainment." In Proceedings of the 2006 Winter Simulation Conference, edited by I. F. Perrone, F. P. Wieland, J. Liu, B. G. Lawson, D. M. Nicol, and R. M. Fujimoto, 1324-1331. Piscataway, New Jersey: Institute of Electrical and Electronics Engineers, Inc.

Smoveland, C., D. Uyeno, I. Vertinsky, and R. Vickson. 1973. “Ambulance Location: A Probabilistic Enumeration Approach,” Management Science, 20, 686-698.

Snyder, L.V. 2006. "Facility Location Under Uncertainty: A Review," IIE Transactions, 38(7): 537-554.

Vincent, P. and I. Rubin. 2004. "A Framework and Analysis for Cooperative Search Using UAV Swarms." Proceedings of the 2004 Association for Computing Machinery (ACM) Conference, 79-86, Nicosia, Cyprus, ACM.

Volovoi, V. 2006. "Stochastic Petri Nets Modeling using SPN@." Proceedings of the Reliability and Maintainability Symposium (RAMS 2006), 75-81, Newport Beach, California.

Wikimedia Commons, "File: Colorado population map.png." Accessed 30 July 2012. http://commons.wikimedia.org/wiki/File:Colorado_population_map.png.

\section{AUTHOR BIOGRAPHIES}

ERICSON R. DAVIS came to LMI from CACI where he performed research and software development, primarily in the area of image processing. Mr. Davis received his undergraduate degree in mathematics from Washington and Lee University and is working to finish up his master's degree in mathematics from George Mason University. His email address is edavis@lmi.org.

JEREMY M. ECKHAUSE has 15 years' experience developing mathematical optimization and simulation models for transportation, government and defense agencies. Dr. Eckhause has a B.A. in Mathematics from the University of Pennsylvania, an M.S.E. in Operations Research from Princeton University and a Ph.D. in Civil Engineering from the University of Maryland. His email address is jeckhause@lmi.org.

DAVID K. PETERSON is a senior consultant specializing in readiness-based sparing inventory modeling. He earned his Ph.D. in Production and Operations Management from the University of North Carolina in 1987. He is a retired U. S. Air Force logistician. His email address is dapeterson@lmi.org.

MICHAEL R. POUY has over 40 years experience in Operations Research and has also done extensive work in the area of facility location optimization for emergency stockpiles. Mr. Pouy holds a bachelor's degree in Mathematics from Villanova University and a master's degree in Operations Research from George Washington University. His email address is mpouy@lmi.org.

STEPHANIE M. SIGALAS-MARKHAM has 6 years' experience working with inventory simulation and mathematical optimization models for government and defense agencies. Ms Sigalas-Markham has a B.S. in Applied Mathematics from the Georgia Institute of Technology and a M.Sc. in Operations Research from the George Washington University. Her email address is ssigalas@lmi.org.

VITALI VOLOVOI is an independent consultant working for LMI. He holds a Ph.D. in Aerospace Engineering from the Georgia Institute of Technology. His principal fields of expertise are quantitative risk analysis; system safety, reliability, and maintenance modeling and optimization; and stochastic modeling and simulation. His email address is vitalivolovoi@gmail.com. 